\title{
Identifying Risk Factors for MRI-Invisible Prostate Cancer in Patients Undergoing Transperineal Saturation Biopsy
}

\author{
Alberto Artiles Medina (D) \\ Rafael Rodríguez- \\ Patrón Rodríguez \\ Mercedes Ruiz Hernández \\ Marina Mata Alcaraz \\ Silvia García Barreras \\ Guillermo Fernández Conejo \\ Agustín Fraile Poblador \\ Enrique Sanz Mayayo \\ Francisco Javier Burgos Revilla \\ Department of Urology, Hospital \\ Universitario Ramón y Cajal, University \\ of Alcalá, Madrid, Spain
}

Correspondence: Alberto Artiles Medina Department of Urology, Hospital Universitario Ramón y Cajal,

M-607, km. 9, 100, Madrid, 28034, Spain

Tel +91.336 .87 .60$

Fax +91.336.87.59

Email alberc.artiles@gmail.com
Purpose: Prostatic multi-parametric magnetic resonance imaging (mpMRI) has markedly improved the assessment of men with suspected prostate cancer (PCa). Nevertheless, as mpMRI exhibits a high negative predictive value, a negative MRI may represent a diagnostic dilemma. The aim of this study was to evaluate the incidence of positive transperineal saturation biopsy in men who have negative mpMRI and to analyse the factors associated with positive biopsy in this scenario.

Patients and Methods: A retrospective study of men with normal mpMRI and suspicion of PCa who underwent saturation biopsy ( $\geq 20$ cores) was carried out. A total of 580 patients underwent transperineal MRI/transrectal ultrasound fusion targeted biopsies or saturation prostate biopsies from January 2017 to September 2020. Of them, 73 had a pre-biopsy negative mpMRI (with Prostate Imaging - Reporting and Data System, PI-RADS, $\leq 2$ ) and were included in this study. Demographics, clinical characteristics, data regarding biopsy results and potential predictive factors of positive saturation biopsy were collected. Univariate and multivariate logistic regression analyses were used to identify independent risk factors for MRI-invisible PCa. Results: The detection rate of PCa with saturation biopsy in patients with negative MRI was 34/ $73(46.58 \%)$. Out of 34 MRI-invisible prostate cancers detected, 12 (35.29\%) were clinically significant $\mathrm{PCa}$ (csPCa) forms. Regarding factors of positive biopsy, in univariate analysis, the use of 5-alpha reductase inhibitors and free:total prostate-specific antigen (PSA) ratio were associated with the result of the saturation biopsy. In multivariate analysis, only an unfavourable free:total PSA ratio remained a risk factor (OR 11.03, CI95\% 1.93-63.15, p=0.01). Furthermore, multivariate logistic analysis demonstrated that prostate volume $>50 \mathrm{~mL}$ significantly predicts the absence of csPCa on saturation biopsy (OR 0.11, 95\% CI 0.01-0.94, p=0.04).

Conclusion: A free:total PSA ratio $<20 \%$ is a risk factor for MRI-invisible PCa. Saturation biopsy could be considered in patients with suspected $\mathrm{PCa}$, despite having a negative MRI. Keywords: clinically significant prostate cancer, multi-parametric magnetic resonance imaging, negative MRI, prostate cancer, saturation biopsy

\section{Introduction}

Prostatic multi-parametric magnetic resonance imaging (mpMRI) has experienced a great development becoming a cornerstone of the management of prostate cancer (PCa). ${ }^{1}$ It is used to better target lesions in biopsy-naïve patients, before repeating biopsy in patients with a growing prostate-specific antigen (PSA) level and previous negative biopsy, in patients on active surveillance (AS) and to improve the prostate cancer staging, mainly in relation to extracapsular extension. Recently, several guidelines have endorsed 
the routine use of mpMRI in biopsy-naïve patients with suspected $\mathrm{PCa}$ and there is an ongoing implementation process in clinical practice. ${ }^{2}$

The negative predictive value of a normal mpMRI (nMRI) is high, and many urologists may use a nMRI to avoid prostate biopsies altogether. ${ }^{3}$

A meta-analysis showed that the sensitivity and specificity for mpMRI is 0.87 [95\% CI, 0.81-0.91] and 0.68 [95\% CI, 0.56-0.79], respectively. It suggests that mpMRI is a sensitive tool to diagnose PCa. ${ }^{4}$ However, although we live in the era of mpMRI, the role of saturation biopsy in reducing the false negative rate is a controverted issue.

As a consequence of the interest in defining more accurate prostate biopsy schemes, the concepts of "extended biopsy" (10-12 cores) and "saturation biopsy" ( $\geq 20$ cores) have emerged. ${ }^{5}$ Saturation biopsy can be performed via transrectal or transperineal approaches, with similarly high detection rates. Recently, the transperineal approach has been preferred because of sampling accuracy, particularly for the anterior prostate region. The cancer detection rate of transperineal saturation biopsy is approximately $40-60 \%$ in repeated biopsies. Previous studies on saturation repeat biopsy showed higher detection rates than those on TRUS-guided repeat biopsy. ${ }^{6}$

Currently, pre-biopsy multiparametric MRI is recommended by several guidelines, not only for men with persistent suspicion of prostate cancer after prior negative systematic biopsy, but also before the first biopsy. ${ }^{7}$ According to the updated European Association of Urology (EAU) guidelines, in patients with prior negative biopsy, when mpMRI is negative (ie, Prostate Imaging Reporting and Data System, PI-RADS, $\leq 2$ ), and clinical suspicion of prostate cancer is high, there is strong evidence to perform a systematic biopsy based on shared decision-making with the patient. On the other hand, in biopsy-naïve patients, there is a weak evidence to recommend a systematic biopsy when mpMRI is negative, and clinical suspicion of prostate cancer is low. Furthermore, these guidelines recommend to do not use mpMRI as an initial screening tool. ${ }^{8}$

PI-RADS allows standardization of prostate MRI interpretation. The PI-RADS v2 document was launched in 2014. ${ }^{9}$ A considerable variability in PI-RADS score assignment and significant cancer yield across radiologists has been reported. ${ }^{10}$ As a result, scepticism against MRI reporting is prevalent among some clinicians. The study of Nassiri et al, involving more than $2000 \mathrm{MRI} /$ ultrasoundfusion biopsies, indicates that if biopsy is indicated on clinical grounds (eg, palpable abnormality, family or racial history, persistent PSA suspicion), a negative mpMRI should not preclude a systematic biopsy. ${ }^{11}$

With the advent of prostatic mpMRI studies, considered as a reliable diagnostic tool of prostate cancer, it is necessary to identify the predictive factors for positive saturation biopsy in patients with negative mpMRI. Several parameters, especially increased PSA density, may help to select patients who need a rebiopsy after a negative MRI.

MRI-invisible prostate cancer is relatively common. A series of 1042 men undergoing template biopsy regardless of MRI findings, the incidence of csPCa in men with no MRI-suspicious lesions (biopsy-naïve subgroup) was $12 \%$. Furthermore, that percentage increased to $28 \%$ after carefully review of prostatectomy specimens. ${ }^{12}$

The scope of the current study was to assess the incidence of positive transperineal saturation biopsy in men who have a negative mpMRI and to determine the factors associated with positive biopsy in this scenario.

\section{Patients and Methods}

We conducted a retrospective study of men with normal mpMRI and suspected prostate cancer, who underwent saturation biopsy. A total of 580 patients underwent transperineal MRI/TRUS fusion targeted biopsies or saturation prostate biopsies from January 2017 to September 2020 in our centre. Of them, 73 had a pre-biopsy negative mpMRI (with PI-RADS $\leq 2$ ) and were included in this study.

All MRI studies were interpreted by experienced radiologists using the Prostate Imaging Reporting and Data System (PI-RADS version 2) and they were re-read before biopsy. Saturation biopsy consisted of at least 20 biopsy cores taken from the prostate and it was performed using the transperineal approach. All biopsies were performed by one urologist with extensive experience in prostate cancer diagnosis.

Patient records were reviewed, and demographic and clinical data were collected. Additionally, we investigated the potential predictive factors of positive saturation biopsy in MRI-negative men: Body mass index (BMI), prostate cancer family history of first-degree relatives, PSA level, PSA-density and PSA-velocity, abnormal findings on digital rectal examination (DRE), previous biopsy showing high-grade prostatic intraepithelial neoplasia (HGPIN) or atypical small acinar proliferation (ASAP), prostate volume measured by MRI, number of previous biopsies, number of biopsy cores, 
and indicators of systemic inflammation, such as neutrophil-lymphocyte ratio (NLR), platelet-to-lymphocyte ratio (PLR) and neutrophil-to-monocyte ratio (NMR). Systemic immune response index (SII) was calculated as follows: SII $=$ platelet $\times$ neutrophil-to-lymphocyte ratio. Clinically insignificant prostate cancer was defined as Gleason score $<7, \leq 3$ positive cores and $\leq 50 \%$ core involvement.

Continuous variables were expressed as mean and standard deviation (SD), and categorical variables were expressed as frequency counts and percentages. The Shapiro-Wilk test was used to assess the normality of the distribution of continuous variables. Statistical tests used to determine the association between some factors and positive saturation biopsy included chi-square test or Fisher's exact test for categorical variables and Student's $t$-test or Wilcoxon's rank sum test for continuous variables. Multivariate logistic regression analyses were also conducted to assess the factors associated with the positive saturation biopsy. A two-tailed p-value of less than 0.05 was considered as statistically significant. All statistical analyses were performed using STATA 14 (StataCorp, College Station, Texas, USA).

\section{Results}

Seventy-three saturation biopsies were performed during the study period. The incidence of prostate cancer was 34/ $73(46.58 \%)$ in men with nMRI who underwent a saturation biopsy. Twenty-two out of 34 (64.71\%) had a clinically insignificant prostate cancer. Seven patients enrolled in AS (19.44\%) with prior negative mpMRI were upgraded after saturation biopsy. Table 1 shows the tumour characteristics of patients with positive saturation biopsy and negative MRI.

Mean age was similar in both groups (62.89 years - SD 6.47 - in the positive saturation biopsy vs 65.08 years SD $6.78-$ in the normal biopsy group, $p=0.16$ ). $84.62 \%$ of patients with both no prior negative biopsy and free:total PSA $<20 \%$ had a positive saturation biopsy (11/13).

Table 2 contains the baseline characteristics of MRInegative patients with positive and negative saturation biopsy. Regarding baseline characteristics, we found statistically significant differences in the use of 5-alpha reductase inhibitors, prostate volume, PSA range and free:total PSA ratio. In univariate analysis (see Table 3), the use of 5-alpha reductase inhibitors and free:total PSA ratio were associated with the result of saturation biopsy. In the multivariate analysis, only an unfavourable free:
Table I Tumour Characteristics in Patients with Positive Saturation Biopsy and Prior Negative MRI $(n=34)$

\begin{tabular}{|c|c|}
\hline Variable & Value \\
\hline \multicolumn{2}{|l|}{ Grade } \\
\hline Gleason $6(3+3)$ (ISUP I) & 22 (64.7I\%) \\
\hline Gleason 7 (3+4) (ISUP 2) & $8(23.52 \%)$ \\
\hline Gleason 7 (4+3) (ISUP 3) & I (2.94\%) \\
\hline Gleason 8 (4+4) (ISUP 4) & $2(5.88 \%)$ \\
\hline Gleason 9 (4+5) (ISUP 5) & I (2.94\%) \\
\hline Percentage of positive cores & 12.89 (SD I I.38) \\
\hline Extent of involvement of needle cores & I0.4I (SD 9.4I) \\
\hline Bilateral tumor & II (33.33\%) \\
\hline $\mathrm{csPCa}$ & $12(35.29 \%)$ \\
\hline Gleason score in active surveillance patients $(n=36)$ & \\
\hline Gleason 6 & $11(30.55 \%)$ \\
\hline Gleason 7 or greater & 7 (19.44\%) \\
\hline
\end{tabular}

Abbreviation: csPCa, clinically significant prostate cancer.

total PSA ratio remained significant independent predictor of a positive saturation biopsy (OR 11.03, CI95\% 1.93$63.15, \mathrm{p}=0.01$ ).

Out of 34 MRI-invisible cancers detected, 12 (35.29\%) patients had a clinically significant prostate cancer (csPCa). For the purpose of determining the factors associated with a csPCa on saturation biopsy, patients were divided into 2 groups according to the result of saturation biopsy: The csPCa patients group $(\mathrm{n}=12)$ and the group composed of patients with normal saturation biopsy or with an insignificant PCa $(\mathrm{n}=61)$ (Table 4). With regard to the baseline characteristics of patients diagnosed with csPCa, a statistically significant difference between groups was found in prostate volume and PSA density. On univariate analysis of risk factors for csPCa, only prostate volumen $>50 \mathrm{~mL}$ was associated with the presence of csPCa on core biopsy samples. Finally, multivariate analysis showed that prostate volume $>50 \mathrm{~mL}$ significantly predicts absence of csPCa on saturation biopsy (OR 0.11, 95\% CI 0.01-0.94, $\mathrm{p}=0.04$ ) (Table 5).

\section{Discussion}

Prostate mpMRI has become a promising tool used to improve the accuracy of prostate cancer detection. Until recently, saturation biopsy was considered the method of choice to improve prostate cancer-detection rate after previous negative biopsy series. ${ }^{13}$ It is important to define the role of the saturation biopsy in the era of mpMRI. 
Table 2 Baseline Characteristics of MRI-Negative Patients with Positive and Negative Saturation Biopsy

\begin{tabular}{|c|c|c|c|}
\hline \multirow[t]{2}{*}{ Variable } & \multicolumn{2}{|c|}{ Biopsy Outcome } & \multirow[t]{2}{*}{$\mathbf{p}$} \\
\hline & Negative $(n=39)$ & Positive (Gleason $\geq 6)(n=34)$ & \\
\hline Age & 62.89 (SD 6.47) & 65.08 (SD 6.78) & 0.16 \\
\hline BMI & 27.57 (SD 3.38) & 27.93 (SD 4.39) & 0.81 \\
\hline Suspicious DRE & $2(5.26 \%)$ & $2(5.88 \%)$ & I \\
\hline Family history of prostate cancer & $\mathrm{I}(2.63 \%)$ & 0 & 1 \\
\hline Use of 5-alpha reductase inhibitors & 7 (23.33\%) & I (2.94\%) & $0.02 *$ \\
\hline Prior ASAP & $2(5.13 \%)$ & $3(8.82 \%)$ & 0.66 \\
\hline Prior HG-PIN & I (2.63\%) & 0 & I \\
\hline Active surveillance program & $18(47.37 \%)$ & $18(52.94 \%)$ & 0.64 \\
\hline Prostate volume & 69.45 (SD 33.63) & 54.49 (SD 30.14) & $0.03 *$ \\
\hline Prior negative biopsy (at least one) & $18(51.43 \%)$ & $10(31.25 \%)$ & 0.09 \\
\hline Number of prior negative biopsies & 0.88 (SD I.05) & $0.46(S D 0.80)$ & 0.07 \\
\hline PSA range & & & $0.03^{*}$ \\
\hline$<10$ & $24(64.86 \%)$ & $29(87.88 \%)$ & \\
\hline$\geq 10$ & $13(35.14 \%)$ & $4(12.12 \%)$ & \\
\hline PSA level & 10.34 (SD 9.37) & 7.55 (SD 3.20) & 0.53 \\
\hline PSA density & $0.15(S D 0.13)$ & 0.16 (SD 0.09) & 0.32 \\
\hline Free:total PSA ratio & $0.22(\mathrm{SD} 0.08)$ & $0.16(\mathrm{SD} 0.07)$ & $0.01 *$ \\
\hline Intervals of free:total PSA ratio & & & $0.02 *$ \\
\hline$<20 \%$ & $6(37.50 \%)$ & 17 (80.95\%) & \\
\hline$\geq 20 \%$ & $10(62.50 \%)$ & $4(19.05 \%)$ & \\
\hline PSA velocity & 4.31 (SD 6.24) & 1.72 (SD 0.95) & 0.45 \\
\hline Total biopsy cores & 23.18 (SD 4.4I) & 21.38 (SD 4.04) & 0.10 \\
\hline NLR & $1.90(\mathrm{SD} 0.79)$ & $1.92(S D 0.70)$ & 0.74 \\
\hline PLR & 94.31 (SD 29.34) & 102.84 (SD 27.25) & 0.18 \\
\hline NMR & 7.43 (SD I.94) & 7.22 (SD 2.29) & 0.42 \\
\hline SII & 400593.6 (SD 161906.2) & 397579.9 (SD I47953) & 0.93 \\
\hline
\end{tabular}

Note: Asterisks $(*)$ represent statistical significance.

Abbreviations: BMI, body mass index; DRE, digital rectal examination; HG-PIN, high-grade prostatic intraepithelial neoplasia; ASAP, atypical small acinar proliferation; NLR, neutrophil-lymphocyte ratio; PLR, platelet-to-lymphocyte ratio; NMR, neutrophil-to-monocyte ratio; SII, systemic immune response index.

Men with a persistent suspicion of PCa with nMRI represents a diagnostic dilemma for urologists because of the high negative predictive value (NPV) of MRI for significant prostate cancer. ${ }^{14}$ The overall estimated false negative rate of mpMRI is around $10-20 \% .{ }^{15}$ The reported false-negative rates of csPCa for targeted fusion prostate biopsy of PI-RADS $\geq 3$ and $\geq 4$ lesions are $16.2 \%$ and $39.7 \%$, respectively. ${ }^{16}$ Therefore, it is important to address the need for biopsy in these patients and to evaluate different predictive factors of positive saturation biopsy in this scenario.

The concept of "mpMRI-invisible disease" has been introduced. MRI-visible tumors are associated with underexpression of cellular organization genes. Mutations in known cancer-associated genes, for example PTEN, could explain the phenomenon of mpMRI-invisible cancer. ${ }^{17}$
Buisset et al analysed the risk of csPCa in a nMRI biopsy-naïve population (503 patients) at baseline and during long-term follow-up. They reported that the risk of csPCa of this population was 6-9\% at baseline (systematic 12-core biopsy) and 7-13\% at long-term follow-up depending on csPCa definitions. Furthermore, they found that PSA density (PSAd) $\geq 0.15 \mathrm{ng} / \mathrm{mL} / \mathrm{mL}$, clinical stage $\geq \mathrm{T} 2 \mathrm{a}$ and $\mathrm{PCa}$-family-history were predictors of csPCa risk. ${ }^{18}$ Our study confirms the importance of performing a biopsy in negative-MRI men suspected of having prostate cancer. In our study, the rate of csPCa is $12 / 73$ $(16.4 \%)$. Overall, prostate cancer was detected in 34/73 (46.58\%) patients. Although we always brought into focus the clinically significant cancer, some Gleason 6 cancers may have the biological potential for de-differentiation and so they require follow-up. ${ }^{12}$ 
Table 3 Univariate and Multivariate Analysis of Factors Related to the Positive Saturation Biopsy

\begin{tabular}{|c|c|c|c|c|}
\hline \multirow[t]{2}{*}{ Variable } & \multicolumn{2}{|c|}{ Univariate } & \multicolumn{2}{|c|}{ Multivariate } \\
\hline & Odds Ratio (Cl) & $\mathbf{p}$ & Odds Ratio (CI) & $\mathbf{p}$ \\
\hline Age & $1.05(0.98-1.13)$ & 0.16 & - & - \\
\hline BMI & $1.02(0.90-1.16)$ & 0.71 & - & - \\
\hline Suspicious DRE & $1.12(0.15-8.45)$ & 0.91 & - & - \\
\hline Family history of prostate cancer & - & - & - & - \\
\hline Use of 5-alpha reductase inhibitors & $0.09(0.01-0.86)$ & $0.03^{*}$ & - & - \\
\hline Prior ASAP & $1.79(0.28-11.4)$ & 0.54 & - & - \\
\hline Prior HG-PIN & - & - & - & - \\
\hline Active surveillance program & $1.25(0.49-3.16)$ & 0.64 & - & - \\
\hline Prostate volumen $>50 \mathrm{~mL}$ & $0.49(0.19-1.28)$ & 0.15 & $1.71(0.29-10.07)$ & 0.55 \\
\hline Prior negative biopsy (at least one) & $0.43(0.16-1.16)$ & 0.09 & $4.48(0.39-50.39)$ & 0.22 \\
\hline PSA level & $0.93(0.85-1.02)$ & 0.13 & $0.70(0.4 I-I .2 I)$ & 0.20 \\
\hline PSA density & $1.57(0.03-84.8 \mathrm{I})$ & 0.82 & - & - \\
\hline Free:total PSA ratio $<20 \%$ & $9.35(2.04-42.65)$ & $0.00 *$ & $11.03(1.93-63.15)$ & $0.0 I^{*}$ \\
\hline PSA velocity & $0.77(0.49-1.19)$ & 0.24 & - & - \\
\hline Total biopsy cores & $0.90(0.80-1.01)$ & 0.08 & - & - \\
\hline NLR & $1.02(0.55-1.93)$ & 0.93 & - & - \\
\hline PLR & $1.01(0.99-1.03)$ & 0.21 & - & - \\
\hline NMR & $0.95(0.76-1.19)$ & 0.68 & - & - \\
\hline SII & $0.99(0.99-1.00)$ & 0.93 & - & - \\
\hline
\end{tabular}

Note: Asterisks $(*)$ represent statistical significance.

Abbreviations: BMI, body mass index; DRE, digital rectal examination; HG-PIN, high-grade prostatic intraepithelial neoplasia; ASAP, atypical small acinar proliferation; NLR, neutrophil-lymphocyte ratio; PLR, platelet-to-lymphocyte ratio; NMR, neutrophil-to-monocyte ratio; SIl, systemic immune response index.

The influence of the number of cores obtained in the detection rate of $\mathrm{PCa}$ has been extensively addressed in the literature. An increase of $\mathrm{PCa}$ detection rate of $5-20 \%$ has been reported when more than six cores are taken at prostate biopsy. ${ }^{19}$ Pepe et al analysed the number of needle cores combined with mpMRI needed to diagnose all csPCa in men who undergo transperineal saturation biopsy. They concluded that a maximum of 20 systematic transperineal needle cores could detect all cases of csPCa and reduce the diagnosis of insignificant PCa. ${ }^{20}$ In our study, we collected data about total number of biopsy cores. However, the number of biopsy cores was similar between both groups.

As many authors have pointed out, a negative MRI does not guarantee the absence of significant prostate cancer. Some reports have shown alarming rates of $\mathrm{PCa}$ among patients with nMRI. For example, Branger et al retrospectively analysed patients who underwent radical prostatectomy and who had a preoperative negative mpMRI. Final pathology showed that $16.9 \%$ had extraprostatic extension, $13.8 \%$ had primary Gleason pattern $4(4+$ 3 and above), and $47.5 \%$ had secondary Gleason pattern 4 or 5. Furthermore, they indicated that data are especially sensitive to radiologist experience. ${ }^{21}$ Similarly, Kim et al have reported that among a total of 196 negative preoperative mpMRI patients who underwent radical prostatectomy, $59.7 \%$ had Gleason score $3+4$ and $22.4 \%$ had Gleason score $\geq 4+3 .^{22}$ A previous report of Sokhi indicates that ISUP $\geq 2$ cancer detection rates were $8 \%$ (5/61) for PI-RADS $1-2 .^{23}$

Recently, a systematic review including a total of sixteen studies was carried out by Pagniez et al. The aim was to determine the predictive ability of some factors (age, prostate specific antigen, prostate specific antigen isoforms, prostate specific antigen density, PCA3, prostate volume, family history, ethnicity and risk calculators) for clinically significant prostate cancer diagnosis after prebiopsy negative magnetic resonance imaging in prostate cancer naïve patients. PSA density was the best studied and the strongest predictor of csPCa in patients with prebiopsy nMRI. Furthermore, they conclude that the use of PSA density less than $0.15 \mathrm{ng} / \mathrm{mL} / \mathrm{mL}$ in patients with prebiopsy nMRI was the most useful factor to identify men without csPCa, in whom biopsy could be precluded. ${ }^{24}$ Similarly, in our study we investigated different potential predictors of positive biopsy in patients with nMRI and who underwent saturation biopsy. We observed that an unfavourable free:total PSA ratio was a significant independent predictor of a positive saturation biopsy and 
Table 4 Baseline Characteristics of Patients Diagnosed with csPCa

\begin{tabular}{|c|c|c|c|}
\hline \multirow[t]{2}{*}{ Variable } & \multicolumn{2}{|c|}{ Biopsy Outcome } & \multirow[t]{2}{*}{ t } \\
\hline & $\begin{array}{l}\text { Gleason } 6 \text { or Negative Biopsy } \\
\qquad(n=61)\end{array}$ & $\begin{array}{l}\text { Clinically Significant Prostate Cancer } \\
\qquad(n=12)\end{array}$ & \\
\hline Age & 63.40 (SD 6.56) & 66.5 (SD 6.88) & 0.14 \\
\hline BMI & $27.63($ SD 4.00$)$ & $28.23($ SD 3.4I) & 0.58 \\
\hline Suspicious DRE & $4(6.67 \%)$ & 0 & 1 \\
\hline Family history of prostate cancer & I (I.69\%) & 0 & 1 \\
\hline Use of 5-alpha reductase inhibitors & $8(15.38 \%)$ & 0 & 0.33 \\
\hline Prior ASAP & $4(6.56 \%)$ & I (8.33\%) & 1 \\
\hline Prior HG-PIN & I (I.67\%) & 0 & 1 \\
\hline Active surveillance program & $29(48.33 \%)$ & $7(58.33 \%)$ & 0.53 \\
\hline Prostate volume & $66.26(S D 33.96)$ & $41.72(S D \mid 0.95)$ & $0.02 *$ \\
\hline Prior negative biopsy (at least one) & $26(46.43 \%)$ & $2(18.18 \%)$ & 0.10 \\
\hline Number of prior negative biopsies & $0.73(\mathrm{SD} 0.94)$ & 0.45 (SD I.03) & 0.18 \\
\hline PSA range & & & 0.49 \\
\hline$<10$ & $44(75.86 \%)$ & $8(66.67 \%)$ & \\
\hline$\geq 10$ & $14(24.14 \%)$ & $4(33.33 \%)$ & \\
\hline PSA level & 9.13 (SD 7.70) & 8.52 (SD 4.62) & 0.94 \\
\hline PSA density & $0.15(S D 0.11)$ & $0.21(S D 0.11)$ & $0.02 *$ \\
\hline Free:total PSA ratio & 0.20 (SD 0.08) & 0.14 (SD 0.05) & 0.12 \\
\hline Intervals of free:total PSA ratio & & & 0.07 \\
\hline$<20 \%$ & $17(53.12 \%)$ & 5 (100\%) & \\
\hline$\geq 20 \%$ & 15 (46.88\%) & 0 & \\
\hline PSA velocity & 3.28 (SD 5.02) & $\mathrm{I} .77$ (SD 0.8I) & 0.96 \\
\hline Total biopsy cores & 22.25 (SD 4.57) & 22.75 (SD 2.66) & 0.66 \\
\hline NLR & $1.90(S D 0.79)$ & 1.96 (SD 0.49) & 0.43 \\
\hline PLR & 96.70 (SD 27.67) & $105.29($ SD 32.86) & 0.37 \\
\hline NMR & 7.33 (SD 2.09) & 7.12 (SD 2.08) & 0.97 \\
\hline SII & 396970.6 (SD I59665) & 410369.9 (SD I32969.6) & 0.62 \\
\hline
\end{tabular}

Note: Asterisks $(*)$ represent statistical significance.

Abbreviations: BMI, body mass index; DRE, digital rectal examination; HG-PIN, high-grade prostatic intraepithelial neoplasia; ASAP, atypical small acinar proliferation; NLR, neutrophil-lymphocyte ratio; PLR, platelet-to-lymphocyte ratio; NMR, neutrophil-to-monocyte ratio; SII, systemic immune response index.

a large prostate volume $(>50 \mathrm{~mL})$ can predict the absence of csPCa. However, we focused on saturation biopsy technique.

PSAd has been shown to be a predictor of csPCa and disease progression on AS for men with low-risk PCa. The combination of saturation biopsy and mpMRI at confirmatory and repeated evaluation can reduce the reclassification rate during AS follow-up (only 5.4\% at 3 years). ${ }^{25}$ According to Roscigno et al, PSAd $\geq 0.20 \mathrm{ng} / \mathrm{mL}^{2}$ is a valuable tool for improving the accuracy of mpMRI results for reclassification of patients in $\mathrm{AS}^{26}$

In the present study we sought to define a group with lower risk for PCa among patients with nMRI. Likewise, Oishi et al proposed that a subset of men with negative MRI, density of PSA less than $0.15 \mathrm{ng} / \mathrm{mL} / \mathrm{cc}$ and prior negative biopsy may safely avoid rebiopsy. Stonier et al state that the sensitivity and NPV of transrectal and transperineal biopsy increased to $96.6 \%$ and $90.6 \%$, respectively, when a PSA density threshold of $0.15 \mathrm{ng} / \mathrm{mL} / \mathrm{mL}$ was used in nMRI (scores 1-2). ${ }^{27}$ Furthermore, Kuhlmann et al identified black race and PSA density as risk factors for the presence of nMRI prostate cancer. ${ }^{15}$ Our work indicates that an independent risk factor for csPCa is a smaller prostate gland volume.

Previous work has focused on the value of indicators of cancer-related inflammation, such as PLR and NLR, in $\mathrm{PCa}$ detection. Adhyatma et al evaluated pre-biopsy values of PLR and NLR in predicting PCa and concluded that NLR could be useful. ${ }^{28}$ Furthermore, SII and SII combination with PI-RADS score have been reported as 
Table 5 Univariate and Multivariate Analysis of Factors for the Detection of Clinically Significant Prostate Cancer

\begin{tabular}{|c|c|c|c|c|}
\hline \multirow[t]{2}{*}{ Variable } & \multicolumn{2}{|c|}{ Univariate } & \multicolumn{2}{|c|}{ Multivariate } \\
\hline & Odds Ratio (Cl) & $\mathbf{p}$ & Odds Ratio (Cl) & $\mathbf{p}$ \\
\hline Age & $1.07(0.97-1.17)$ & 0.14 & - & - \\
\hline BMI & $1.04(0.88-1.22)$ & 0.62 & - & - \\
\hline Suspicious DRE & - & - & - & - \\
\hline Family history of prostate cancer & - & - & - & - \\
\hline Use of 5-alpha reductase inhibitors & - & - & - & - \\
\hline Prior ASAP & $1.29(0.13-12.72)$ & 0.82 & - & - \\
\hline Prior HG-PIN & - & - & - & - \\
\hline Active surveillance program & $1.49(0.43-5.24)$ & 0.53 & - & - \\
\hline Prostate volumen $>50 \mathrm{~mL}$ & $0.18(0.04-0.94)$ & $0.04 *$ & $0.11(0.01-0.94)$ & $0.04 *$ \\
\hline Prior negative biopsy (at least one) & $0.25(0.05-1.29)$ & 0.10 & $0.48(0.08-2.73)$ & 0.41 \\
\hline PSA level & $0.98(0.89-1.08)$ & 0.79 & - & - \\
\hline PSA density & $38.21(0.36-4023.70)$ & 0.12 & - & - \\
\hline Free:total PSA ratio & $2.72 \mathrm{e}^{-06}\left(1.20^{\mathrm{e}-13}-61.62\right)$ & 0.14 & - & - \\
\hline PSA velocity & $0.85(0.52-1.39)$ & 0.52 & - & - \\
\hline Total biopsy cores & $1.03(0.88-1.19)$ & 0.71 & - & - \\
\hline NLR & I.I2 (0.49-2.52) & 0.78 & - & - \\
\hline PLR & I.0I (0.99-I.03) & 0.34 & - & - \\
\hline NMR & $0.94(0.69-1.28)$ & 0.69 & - & - \\
\hline SII & $1.00(0.99-1.00)$ & 0.78 & - & - \\
\hline
\end{tabular}

Note: Asterisks $(*)$ represent statistical significance.

Abbreviations: BMI, body mass index; DRE, digital rectal examination; HG-PIN, high-grade prostatic intraepithelial neoplasia; ASAP, atypical small acinar proliferation; NLR, neutrophil-lymphocyte ratio; PLR, platelet-to-lymphocyte ratio; NMR, neutrophil-to-monocyte ratio; SIl, systemic immune response index.

a significant diagnostic marker in patients with high-grade PCa (ISUP grade 3-5). ${ }^{29}$ In contrast to these earlier findings, we did not find association between these parameters and the result of saturation biopsy.

Some controversy remains as to whether patients with prostate cancer suspicion and negative MRI can safely omit biopsy. Regis et al have stated categorically that PIRADS 2 should not be considered as a negative MRI because its NPV in biopsy-naïve patients was $84 \% .{ }^{30}$ In consonance with this author, Ma et al considered that only PI-RADS 1 has a high NPV. ${ }^{31}$

To sum up, there is no consensus on which patients with a negative prostate MRI should forego biopsy. According to the majority of guidelines, including those published from American Urological Association (AUA), European Association of Urology (EAU), and National Comprehensive Cancer Network (NCCN), men with an elevated PSA and a negative MRI are referred for biopsy. Some authors defend that biopsy could be avoided in the absence of high-risk features, such as clinical suspicion, family history, low PSA density, and those with low scores on the $4 \mathrm{~K}$ or Prostate Health Index (PHI), and PCA3 assays. $^{32}$
A nMRI does not necessarily exclude the possibility of a csPCa. Our findings highlight the need for biopsy in men with negative MRI and a free:total PSA ratio $<0.2$, and indicate that saturation biopsy can play a relevant role in detecting prostate cancer in this context. Furthermore, a large prostate volume $(>50 \mathrm{~mL})$ can predict the absence of csPCa on saturation biopsy sample in MRI-negative patients. The most important limitations of our work lie in the small sample size and in the retrospective study design. Furthermore, in this study we did not include some parameters which could be potentially useful, such as proPSA (included in the PHI) or PCA3.

\section{Abbreviations}

AS, active surveillance; ASAP, atypical small acinar proliferation; BMI, body mass index; csPCa, clinically significant prostate cancer; DRE, digital rectal examination; HG-PIN, high-grade prostatic intraepithelial neoplasia; mpMRI, multi-parametric magnetic resonance imaging; NLR, neutrophil-lymphocyte ratio; NMR, neutrophil-tomonocyte ratio; nMRI, normal mpMRI; PCa, prostate cancer; PLR, platelet-to-lymphocyte ratio; SII, systemic immune response index; TRUS, transrectal ultrasound. 


\section{Ethics Approval and Informed Consent}

This research is retrospective and involving the collection of existing data and records. All procedures performed in studies involving human participants were in accordance with the ethical standards of the institutional and/or national research committee(s) and with the Helsinki Declaration. The ethical committee of Hospital Ramón y Cajal of Madrid approved all procedures of the study and also provided the waiver for the written informed consent.

\section{Author Contributions}

All authors contributed to data analysis, drafting or revising the article, have agreed on the journal to which the article will be submitted, gave final approval of the version to be published, and agree to be accountable for all aspects of the work.

\section{Disclosure}

All authors declare that they have no conflicts of interest in this work.

\section{References}

1. Catalá V, Vilanova JC, Gaya JM, et al. Multiparametric magnetic resonance imaging and prostate cancer: what's new? Radiol. 2017;59 (3):196-208. doi:10.1016/j.rxeng.2017.03.003

2. Papoutsaki M-V, Allen C, Giganti F, et al. Standardisation of prostate multiparametric MRI across a hospital network: a London experience. Insights into Imaging. 2021;12(1):1-11. doi:10.1186/ S13244-021-00990-Y

3. Frydenberg M. Reliability of negative multiparametric MRI of the prostate: can we avoid the biopsy? Not yet BJU Int. 2017;119(5):E9E10. doi:10.1111/bju.13787

4. Zhen L, Liu X, Yegang C, et al. Accuracy of multiparametric magnetic resonance imaging for diagnosing prostate Cancer: a systematic review and meta-analysis. BMC Cancer. 2019;19(1):1-5. doi:10.1186/s12885-019-6434-2

5. Maccagnano C, Gallina A, Roscigno M, et al. Prostate saturation biopsy following a first negative biopsy: state of the art. Urol Int. 2012;89(2):126-135. doi:10.1159/000339521

6. Nakai Y, Tanaka N, Anai S, et al. Transperineal template-guided saturation biopsy aimed at sampling one core for each milliliter of prostate volume: 103 cases requiring repeat prostate biopsy. $B M C$ Urol. 2017;17(1):1-6. doi:10.1186/s12894-017-0219-1

7. Schoots IG, Padhani AR. Delivering Clinical impacts of the MRI diagnostic pathway in prostate cancer diagnosis. Abdom Radiol. 2020:1-1. doi:10.1007/s00261-020-02547-x

8. EAU guidelines: prostate cancer uroweb. Available from: https:// uroweb.org/guideline/prostate-cancer/?type=summary-of-changes. Accessed November 22, 2020.

9. Barrett T, Turkbey B, Choyke PL. PI-RADS version 2: what you need to know. Clin Radiol. 2015;70(11):1165-1176. doi:10.1016/j. crad.2015.06.093

10. Sonn GA, Fan RE, Ghanouni P, et al. Prostate magnetic resonance imaging interpretation varies substantially across radiologists. Eur Urol Focus. 2019;5(4):592-599. doi:10.1016/j.euf.2017.11.010
11. Nassiri N, Natarajan S, Margolis DJ, et al. Targeted prostate biopsy: lessons learned midst the evolution of a disruptive technology. Urology. 2015;86:432-438. doi:10.1016/j.urology.2015.07.001

12. Marks LS. Some prostate cancers are invisible to magnetic resonance imaging BJU Int. 2016;118(4):492-493. doi:10.1111/bju.13440

13. Dekalo S, Matzkin H, Mabjeesh NJ. High cancer detection rate using cognitive fusion - targeted transperineal prostate biopsies. Int Braz J Urol. 2017;43(4):600-606. doi:10.1590/S1677-5538.IBJU.20 16.0511

14. Itatani R, Namimoto T, Atsuji S, et al. Negative predictive value of multiparametric MRI for prostate cancer detection: outcome of 5-year follow-up in men with negative findings on initial MRI studies. Eur $J$ Radiol. 2014;83(10):1740-1745. doi:10.1016/j. ejrad.2014.06.026

15. Kuhlmann PK, Chen M, Luu M, et al. Patient- and tumor-level risk factors for MRI-invisible prostate cancer. Prostate Cancer Prostatic Dis. 2021:1-8. doi:10.1038/s41391-021-00330-7.

16. Pepe P, Garufi A, Priolo GD, et al. Is it time to perform only magnetic resonance imaging targeted cores? Our experience with 1032 men who underwent prostate biopsy. J Urol. 2018;200(4):774-778. doi:10.1016/J.JURO.2018.04.061

17. Salami SS, Kaplan JB, Nallandhighal S, et al. Biologic significance of magnetic resonance imaging invisibility in localized prostate cancer. JCO Precis Oncol. 2019;3:1-12. doi:10.1200/po.19.00054

18. Buisset J, Norris JM, Puech P, et al. Negative pre-biopsy magnetic resonance imaging and risk of significant prostate cancer: baseline and long-term follow-up results. $J$ Urol. 2020;205:725-731. doi:10.1097/ju.0000000000001414

19. Scattoni V, Maccagnano C, Zanni G, et al. Is extended and saturation biopsy necessary? Int J Urol. 2010;17(5):432-447. doi:10.1111/ J.1442-2042.2010.02479.X

20. Pepe P, Pennisi M, Fraggetta F. How many cores should be obtained during saturation biopsy in the era of multiparametric magnetic resonance? Experience in 875 patients submitted to repeat prostate biopsy. Urology. 2020;137:133-137. doi:10.1016/J.UROLOGY.20 19.11.016

21. Branger N, Maubon T, Traumann M, et al. Is negative multiparametric magnetic resonance imaging really able to exclude significant prostate cancer? The real-life experience. BJU Int. 2017;119 (3):449-455. doi:10.1111/bju. 13657

22. Kim JJ, Byun SS, Lee SE, et al. A negative multiparametric magnetic resonance imaging finding does not guarantee the absence of significant cancer among biopsy-proven prostate cancer patients: a real-life clinical experience. Int Urol Nephrol. 2018;50(11):1989-1997. doi:10.1007/s11255-018-1986-7

23. Sokhi HK, Padhani AR, Patel S, et al. Diagnostic yields in patients with suspected prostate cancer undergoing MRI as the first-line investigation in routine practice. Clin Radiol. 2020;75(12):950-956. doi:10.1016/j.crad.2020.08.011

24. Pagniez MA, Kasivisvanathan V, Puech P, et al. Predictive factors of missed clinically significant prostate cancers in men with negative magnetic resonance imaging: a systematic review and meta-analysis. J Urol. 2020;204(1):24-32. doi:10.1097/ JU.0000000000000757

25. Pepe P, Pepe L, Pennisi M, Fraggetta F. Which prostate biopsy in men enrolled in active surveillance? Experience in 110 men submitted to scheduled three-years transperineal saturation biopsy combined with fusion targeted cores. Clin Genitourin Cancer. 2021;19:305-308. doi:10.1016/J.CLGC.2021.01.004

26. Roscigno M, Stabile A, Lughezzani G, et al. The use of multiparametric magnetic resonance imaging for follow-up of patients included in active surveillance protocol. can PSA density discriminate patients at different risk of reclassification? Clin Genitourin Cancer. 2020;18(6):e698-e704. doi:10.1016/J. CLGC.2020.04.006 
27. Stonier T, Simson N, Shah T, et al. The "Is mpMRI enough" or IMRIE study: a multicentre evaluation of prebiopsy multiparametric magnetic resonance imaging compared with biopsy. Eur Urol Focus. 2020. doi:10.1016/j.euf.2020.09.012

28. Adhyatma KP, Prapiska FF, Siregar GP, et al. Systemic inflammatory response in predicting prostate cancer: the diagnostic value of neutrophil-to-lymphocyte ratio. Open Access Maced J Med Sci. 2019;7(10):1628-1630. doi:10.3889/OAMJMS.2019.177

29. Sonmez G, Demirtas T, Tombul ST, et al. Diagnostic efficiency of systemic immune-inflammation index in fusion prostate biopsy. Actas Urol Esp. 2021;45(5):359-365. doi:10.1016/J.ACUROE.2021.04.006
30. Regis L, Celma A, Planas J, et al. The role of negative magnetic resonance imaging: can we safely avoid biopsy in P.I.-R.A.D.S. 2 as in P.I.-R.A.D.S. 1? Scand J Urol. 2019;53(1):21-25. doi:10.1080/21681805.2018.1551243

31. Ma HY, Ahmed FS, Luk L, et al. The negative predictive value of a PI-RADS version 2 score of 1 on prostate MRI and the factors associated with a false-negative MRI study. Am J Roentgenol. 2020;215(3):667-672. doi:10.2214/AJR.20.22784

32. The role of magnetic resonance imaging in prostate cancer UpToDate. Available from: https:/www.uptodate.com/contents/therole-of-magnetic-resonance-imaging-in-prostate-cancer/print. Accessed November 20, 2020.

\section{Publish your work in this journal}

Research and Reports in Urology is an international, peer-reviewed, open access journal publishing original research, reports, editorials, reviews and commentaries on all aspects of adult and pediatric urology in the clinic and laboratory including the following topics Pathology, pathophysiology of urological disease; Investigation and treatment of urological disease; Pharmacology of drugs used for the treatment of urological disease. The manuscript management system is completely online and includes a very quick and fair peer-review system, which is all easy to use. Visit http://www.dovepress.com/ testimonials.php to read real quotes from published authors. 